\title{
Dose escalation of second-line sunitinib results in rapid partial remission of multiple hepatic metastases
}

\author{
Catherine Guevremont, BSc; Florin I. Mija, MD; Hendrik Isbarn, MD; Claudio Jeldres, MD; Giovanni Lughezzani, \\ MD; Maxine Sun, MD; Pascale Audet, MD; Paul Perrotte, MD; Pierre I. Karakiewicz, MD, FRCSC
}

\begin{abstract}
A 58-year-old man with metastatic clear cell renal cell carcinoma on sunitinib therapy, who previously failed on sorafenib, was found to have progression of multiple hepatic metastases; he was on a standard sunitinib dose of $50 \mathrm{mg}$ /day (4 weeks on, 2 weeks off). Due to the unavailability of alternative therapies, a sunitinib dose escalation of $50 \mathrm{mg} /$ day was attempted. After one 6-week cycle of continuously dosed sunitinib $50 \mathrm{mg}$, the hepatic lesions regressed. After the second cycle, virtual disappearance of the lesions was recorded. There was no added toxicity. These findings suggest that sunitinib dose escalation to $50 \mathrm{mg} /$ day using continuous daily administration dosing might represent a valid, effective and well-tolerated therapeutic option in patients who progress on standard sunitinib therapy.
\end{abstract}

Can Urol Assoc J 2009;3(6):E92-E93

\section{Case report}

A standard sunitinib dose of $50 \mathrm{mg} /$ day (4 weeks on, 2 weeks off) was initiated in a 58-year-old male with metastatic clear cell renal cell carcinoma (lung, liver and retroperitoneal metastases), after rapid disease progression and impending respiratory distress while on sorafenib therapy. Based on the impressive response to sunitinib therapy, a cytonephrectomy was performed 18 months after starting sunitinib. Computed tomography (CT) scans of the abdomen and chest revealed virtually complete resolution of all metastatic lesions (Fig. 1). However, after 24 months of sustained response to standard sunitinib therapy $50 \mathrm{mg} /$ day (4 weeks on, 2 weeks off), CT scans revealed disease progression in the form of multiple hepatic metastases (Fig. 2). The absence of side effects related to sunitinib was also recorded.

Due to the unavailability of alternative treatment regimens and the absence of systemic and hematologic toxicity to the standard sunitinib dose of $50 \mathrm{mg} /$ day (4 weeks on, 2 weeks off), sunitinib dosage of $50 \mathrm{mg} /$ day was administered without the 2-week rest period. After one 6-week cycle of therapy, a significant decrease in the size and number of hepatic metastases was noted (Fig. 2). After the second cycle of continuous dosing of $50 \mathrm{mg} /$ day, a near-complete disappearance of the lesions was seen. No increase in systemic or hematologic toxicity was recorded.

\section{Discussion}

Sunitinib represents the established first-line therapy for patients with locally advanced or metastatic renal cell carcinoma. ${ }^{1}$ Sunitinib is also effective as second-line therapy after sorafenib failure. , 3,4 In the current report, we present a case of a patient with an initial long-term (24 months) and virtually complete response to sunitinib therapy after sorafenib failure. The case is unique because the patient experienced an impressive and extremely rapid response to sunitinib dose escalation to $50 \mathrm{mg} /$ day delivered in continuous daily administration form, as opposed to the conventional dose of $50 \mathrm{mg} /$ day for 4 of 6 weeks dosing on which the patient had a disease relapse. The impressive efficacy of continuously dosed daily sunitinib of $50 \mathrm{mg}$ is even more noteworthy given the lengthy (24 months) previous response to this molecule. Therefore, dose escalation from standard sunitinib dose of $50 \mathrm{mg} /$ day administered for 4 weeks with a rest period of 2 weeks to $50 \mathrm{mg} /$ day continuous dosing may represent a valid alternative to treatment discontinuation with the intent of using a subsequent line therapy. Dose escalation may be particularly important, when the patient has already failed more than one treatment and few therapeutic options remain. Our report also indicates that at least in select patients, dose escalation may be well-tolerated and will not invariably increase the number or the severity of side effects. Our findings are consistent with the reports by Amato and colleagues, which demonstrate increased efficacy of dose-escalated sorafenib versus conventional dosing. 5,6

In this particular case, the patient experienced no additional toxicities, such as fatigue, skin reaction, hypertension, diarrhea or other commonly experienced side effects of sunitinib. However, this is not always the case. Some patients may experience grades I or II toxicity, while others 
Fig. 1. Axial computed tomography (CT) scans of the patient before sunitinib induction therapy, demonstrating the primary renal tumour $\left({ }^{*}\right)$, pleural carcinomitosis of the lung $(A)$ and liver $(\mathrm{C})$ compared to the scans after 18 months of a standard sunitinib dose of 50 $\mathrm{mg} / \mathrm{day}$ (4 weeks on, 2 weeks off) demonstrating virtual resolution of the lung $(B)$ and liver (D) metastases.

A: Axial CT-Scan before sunitinib induction therapy demonstrating pleural carcinomitosis of the lung; B: Axial CT-Scan after 18 months of standard sunitinib demonstrating virtual resolution of the pleural carcinomitosis of the lung; C: Axial CT-Scan before sunitinib induction demonstrating liver and retroperitoneal metastases; D: Axial CT-Scan after 18 months of standard sunitinib and a cytoreductive nephrectomy demonstrating virtual resolution of the liver metastases and removal of the primary tumour.

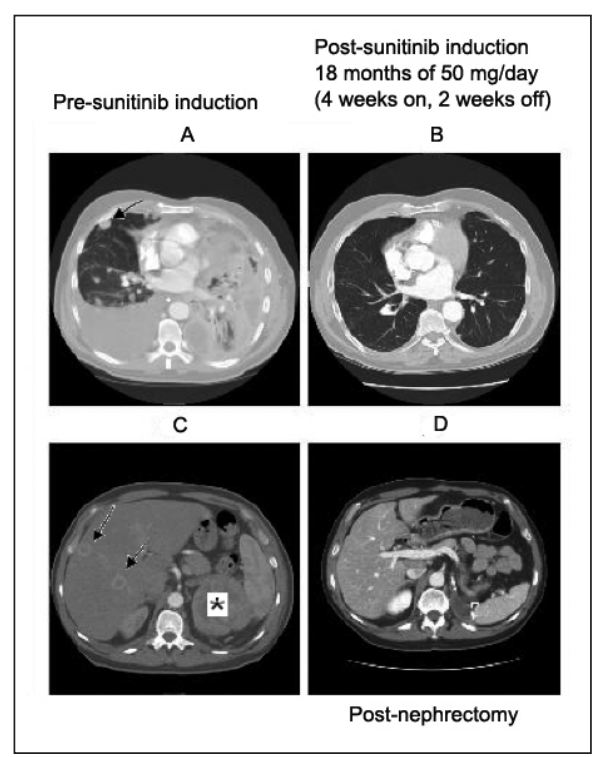

may progress to grades III or IV. Dose escalation can only be considered an option in the absence of grades III or IV toxicity. The presence of grades I or II toxicity should be considered a relative counterindication and requires individual patient counselling. Patients should be aware of the lack of established benefit ratio and the potential increase in the severity of existing side effects or the potential incidence of new side effects. These considerations are particularly important in the context of the noncurative nature of sunitinib therapy.

\section{Conclusion}

Our findings indicate that sunitinib may demonstrate efficacy if its dose is escalated from the standard dosing of $50 \mathrm{mg} /$ day for 4 weeks followed by a 2-week rest period to $50 \mathrm{mg} /$ day administered continuously, after disease progression. It remains to be seen what proportion of sunitinib progressors will respond to sunitinib dose escalation and what proportion of patients tolerant to standard sunitinib dose will develop toxicity with sunitinib dose escalation.

From the Centre Hospitalier de I'Université de Montréal (CHUM), Hôpital Saint-Luc, Montréal, QC

Competing interests: None declared.

This paper has been peer-reviewed.

\section{References}

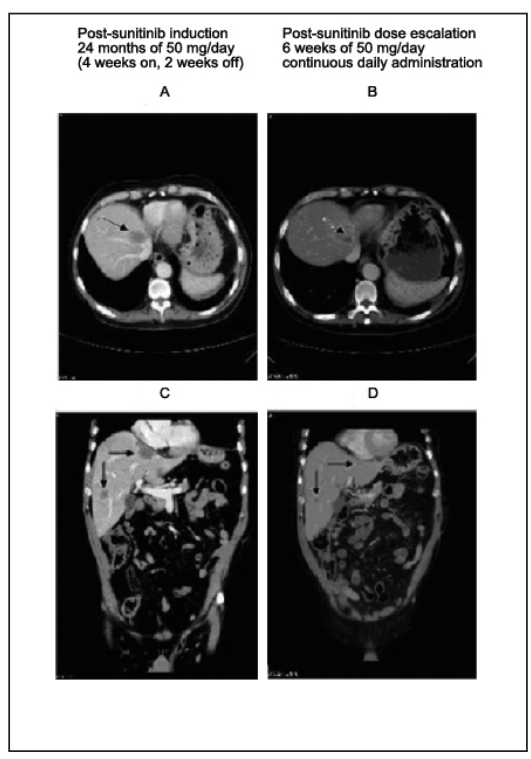

Fig. 2. Axial and coronal computed tomography (CT) scans of the patient after 24 months of a standard sunitinib dose of $50 \mathrm{mg} /$ day (4 weeks on, 2 weeks off) demonstrating hepatic metastases $(\mathrm{A}$ and $\mathrm{C})$ and after one 6-week cycle of sunitinib dose escalation, $50 \mathrm{mg} /$ day delivered in continuous form, demonstrating virtual resolution of hepatic metastases ( $B$ and $D$ ). A: Axial CT-Scan before sunitinib dose-escalation demonstrating the presence of hepatic metastases; B: Axial CT-Scan after 6-week cycle of sunitinib dose escalation demonstrating virtual resolution of hepatic metastases; C: Coronal CT-Scan before sunitinib dose escalation demonstrating the presence of hepatic metastases ; D: Coronal CT-Scan after 6 -week cycle of sunitinib dose escalation demonstrating virtual resolution of hepatic metastases.

1. Motzer RJ, Hutson TE, Tomczak P, et al. Sunitinib versus Interferon Alfa in Metastatic Renal-Cell Carcinoma. N Engl J Med 2007;356:115-24.

2. Dudek AZ, Zolnierek J, Dham A, et al. Sequential Therapy with Sorafenib and Sunitinib in Renal Cell Carcinoma. Cancer 2009;115:61-7.

3. Tamaskar I, Garcia JA, Elson P, et al. Antitumor Effects of Sunitinib or Sorafenib in Patients with Metastatic Renal Carcinoma Who Received Prior Antiangiogenic Therapy, I Urol 2008;179:81-6.

4. Heng DY, Rini BI, Garcia J, et al. Prolonged Complete Responses and Near-Complete Responses to Sunitinib in Metastatic Renal Cell Carcinoma. Clin Genitourin Cancer $2007,5: 446-51$.

5. Amato RJ, Harris P, Dalton M, et al. A phase II trial of intra-patient dose-escalated sorafenib in patients (pts) with metastatic renal cell cancer (MRCC) [abstract]. J Clin Oncol 2007;25:abstr 5026.

6. Amato RJ, Jac J, Harris P, et al. A phase II trial of intra-patient dose-escalated sorafenib in patients (pts) with metastatic renal cell cancer (MRCC). J Clin Oncol 2008;26:abstr 5122.

Correspondence: Dr. Catherine Guevremont, Hôpital Saint-Luc, 1058, rue Saint-Denis, Montréal, QC H2X 3J4; catherine.guevremont@gmail.com 